\title{
Classification of lubricants according to cavitation criteria
}

\author{
Y. Meged, C.H. Venner, W.E. ten Napel \\ The Tribology Group, Department of Mechanical Engineering, University of Twente, Enschede, Netherlands
}

\begin{abstract}
Cavitation in lubrication liquids has long been known to be detrimental to components in hydraulic systems. Damage has been detected in journal bearings, especially under severe dynamic loading, gears, squeeze film dampers and valves.

These findings have led to intensive studies of metal resistance to cavitation erosion, in order to minimize the damage. Results of these studies have been:

(a) classification of known materials according to their resistance

to cavitation erosion;

(b) development of new materials and processes to increase their durability.

One of the main achievements in this respect was the establishment of the ASTM G32-92 Standard Method of Vibratory Cavitation Erosion Test. However, very little was done with respect to the liquid phase, e.g. the lubricants. As a consequence there is no standard procedure for testing of lubricants for their cavitation properties and no relevant specifications in national and international standards.

This study includes theoretical and experimental investigations. The theoretical approach examines the lubricant in elastohydrodynamically lubricated (EHL) contacts. Using numerical simulations, based on Reynolds equation and elastic deformation theory, the pressure profile and film shape have been computed. It is further investigated how the operating conditions affect the properties, e.g. "cavitation energy" of zones of sub-ambient pressure values and if a correlation between these results and cavitation erosion criteria can be found.

The experimental approach includes testing of 20 liquid lubricants, belonging to the following four groups: mineral oils, mineral-based oils, bio degradable oils and synthetic oils. Testing was performed by vibrating a standard aluminium tip in each oil and periodically recording the gravimetric results. These results enabled the classification of the lubricants according to their cavitance, which is inversely proportional to the mass of solid material eroded by a cavitating liquid under controlled conditions.

The results of both approaches can be combined into an engineering tool in the future. This tool may serve the designer to improve the use of existing lubricants and the lubrication industry as an aid for the development of new lubricants with increased cavitance in hydraulic systems.
\end{abstract}

Keywords: Cavitation erosion; Lubricants; Hydraulic systems; Reynolds equation

\section{Introduction}

This study is oriented to the investigation of cavitation erosion phenomena occurring in oil lubricated systems. It includes both theoretical and experimental approaches, whose main goals are:

(a) Establishing a method for the calculation of cavitation erosion potential in operating hydraulic systems.

(b) Classification of commercial liquid lubricants, according to their cavitance.

Rcviewing the tribological literature it appears that until now in theoretical studies of cavitation in lubricated contacts emphasis is on the implications for fluid film formation, rup- ture and reformation. Typical examples of such studies are [1], and [2].

In such works, similar to common practice in consideration of two-phase flows, a modified continuum (Reynolds) equation is derived by including a void fraction of gas (vapour and/or air) into the mass balance. Subsequently, pressure generation is only allowed in those regions where the contact can be fully flooded with the amount of oil locally present. If this can not bc the case without violating mass conservation, the pressure is fixed at ambient pressure and the void fraction of gas in the oil-vapour-air mixture is solved for.

This, for example, applies in the exit region of the contact, but it can also apply in rcgions having an indentation or a 
surface roughness value with a large ratio of depth to width. Such a feature can cause the pressure to drop below the vapour pressure and induce local cavitation.

In the narrowing gap on the downstream side of the feature pressure generation will start again, however, this can only be allowed after verifying that the amount of oil present at such a point is sufficient to fill the gap.

Summarizing, only two possible regimes are distinguished within the film: pressure exceeding ambient in fully flooded zones and ambient pressure in cavitation zones. In these latter cavitated regions with non-vaporous cavitation, flow is assumed to be unidirectional. The most clear example is the exit region of a lubrication contact where the flow is in the form of so-called fingers or streamers.

This approach may indeed have proven very useful for film formation analysis. However, it is not suitable if the purpose of study is to gain insight in the potential danger of cavitation damage. Firstly, the result of applying the above listed algorithm will at most be a "geometrical result", i.e. it will reveal where cavitated regions will occur, and where pressure generation starts. However, no result is obtained as to the potential of such a region for causing damage, let alone that it could be linked to lubrication properties. Secondly, by distinguishing only two sorts of regions, as mentioned above, one region is neglected which is very important when aiming for cavitation damage control.

In this region the pressure drops below the vapour pressure and vapour bubbles will be formed. These vapour bubbles, upon later compression, will implode and may cause damage to the surface. Cavitation damage control calls for linking knowledge of bubble initiation, to the local conditions in the film, under given circumstances.

In the present investigation a first step is set in this direction. In particular, the relation between the magnitude of subambient pressures and operating conditions in a lubricated concentrated contact is investigated. For simplicity the study is restricted to the so-called smooth ElastoHydrodynamically Lubricated (EHL) line contact.

Cavitation investigators have tried to find the properties of the liquids which are dominant in the erosion process and enable rating of the liquids according to their damage potential. Among the properties which were suggested are the density $\rho$ [3], the acoustic impedance $\rho c$ [4] and the viscosity $\eta[5]$.

Relatively few experiments were focused on the properties of lubricating oils. A typical example of such studies was perfonmed by Vercon el al. [6]. In Vercon's work 6 motor oils were tested by means of a magnetostrictive apparatus, vibrating at $20 \mathrm{kHz}$ with an amplitude of $50 \mu \mathrm{m}$. The specimen, made of bearing material, was stationary while the vibrating tip was made of crank shaft steel. One of the main conclusions of this work was that with higher oil viscosity (at $80^{\circ} \mathrm{C}$ ) the erosion intensity increased nearly linearly. This conclusion was supported by experiments carried out at various temperatures in the range of $20-120^{\circ} \mathrm{C}$.
An identical conclusion was reached by Barwell and Scott [7], who tested 26 lubricants, belonging to 10 types, on a 4ball tester. This conclusion was found valid only for lubricants of the same type, but not for all types tested. Barwell concluded that viscosity of the oil did not seem to be a dominant factor in the time taken to pitting failure. This was because some low viscosity fluids gave as long a life as others with much higher viscosities.

As a consequence of these tests it seems necessary to increase the quantity of tested lubricants and types of lubricants. Furthermore, a search for dominant liquid properties in cavitation erosion is recommended.

In the present investigation 20 lubricants, belonging to four types, were tested. The test apparatus was a piezoelectric vibrator, oscillating an aluminium specimen tip at a frequency or $20 \mathrm{kHz}$ and an amplitude of $73 \mu \mathrm{m}$. Gravimetric results were recorded, thus enabling the classification of the lubricants according to their cavitance.

\section{Theoretical approach}

\subsection{Equations}

The contact is modelled using the following three equations. The first is the Reynolds equation for steady state conditions:

$\frac{\partial}{\partial x}\left[\frac{p h^{3}}{\eta}\left(\frac{\partial p}{\partial x}\right)\right]-6 u_{s} \frac{\partial(p h)}{\partial x}=0$

with the boundary conditions $p\left(x_{\mathrm{a}}\right)=p\left(x_{\mathrm{b}}\right)=p_{\mathrm{o}}$, where $p_{\mathrm{o}}$ denotes the ambient pressure. In the present work the assumptions are that $p_{\mathrm{o}}=0$ and that the contact is abundantly supplied with lubricant on all sides.

The density will be assumed to depend on the pressure according to the Dowson and Higginson equation [8] and the Roelands pressure-viscosity relation [9] will be used. This requires some further clarification. Using the Dowson and Higginson equation for the density at pressures below ambient pressure implies that wc assumc the associated density then reflects the presence of vapour bubbles in the oil. Naturally such an assumption can only hold as long as these bubbles are relatively widespread and all together fill only a small fraction of the local volume in the film in the order of $1 \%$.

Under these assumptions the mixture of oil and vapour bubbles still effectively behaves as a single continuum. For the viscosity of the mixture it is assumed that it equals the viscosity of the oil at ambient pressure.

The second equation used is the so-called film thickness equation:

$h(x)=h_{\mathrm{o}}+\frac{x^{2}}{2 R}-\frac{1}{4 \pi E^{\prime}} \int_{x_{0}}^{x_{\mathrm{a}}} p\left(x^{\prime}\right) \ln \left|x-x^{\prime}\right| \mathrm{d} x^{\prime}$ 


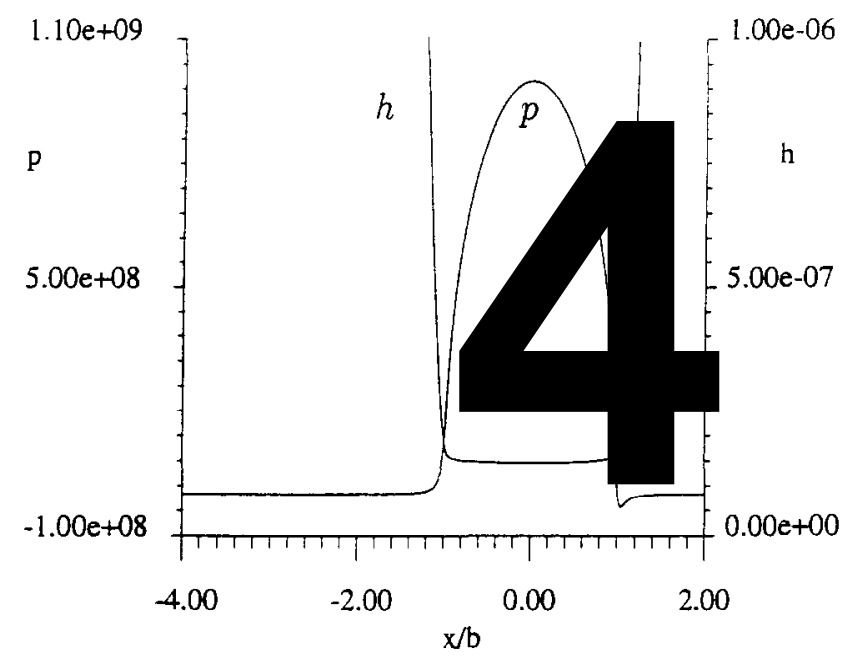

Fig. 1. Pressure $p$ and film thickness $h$ as a function of $x / b$ for the conditions listed in Appendix B.

where $h_{\mathrm{o}}$ is a constant determined by the global condition of force balance:

$w=\int_{x \mathrm{~b}}^{x_{\mathrm{a}}} p\left(x^{\prime}\right) \mathrm{d} x^{\prime}$

\subsection{Results}

After introducing dimensionless variables, based upon the Hertzian dry contact parameters, Eqs. (1)-(3) were discretized on a uniform grid and the resulting discrete system of equations was solved using multigrid techniques. For details with respect to the discrete equations and numerical algorithm the reader is referred to Refs. [10] and [11].

For the present investigation a specific load condition was derived from an actual bearing application. The parameters describing this contact are listed in Appendix B. It is a moderately loaded contact with a maximum Hertzian pressure of $1.0 \mathrm{GPa}$. This Appendix also gives the values of the minimum and central film thickness as obtained from the computations as well as values of the various sets of dimensionless parameters generally used in EHL analysis to characterize load situations.

Fig. 1 shows the pressure and film thickness profiles as computed. The solution has all the characteristics of moderately loaded EHL solutions, see [11].

The film thickness is nearly uniform in the centre and shows a nip in the exit region. The pressure profile is almost semi-elliptic, with the so-called Petrusevich spike preceding the exit region. In this exit region, owing to the imposition of the cavitation condition $p \geq 0$, normally zero (ambient) pressures are found, see $[10,11]$. However, now obviously negative (sub-ambient) pressure values must occur as this cavitation condition was discarded. This can be seen upon enlargement of the pressure profile, i.e. see Fig. 2. This latter figure also shows the dimensionless lubricant density in this region $\bar{\rho}=\rho / \rho_{\mathrm{o}}$. Note that the density deviation associated with the sub-ambient pressures is less than $2 \%$, which justifies the assumption made by using the Dowson and Higginson throughout.

As a next step the surface speed and the reduced radius of curvature of the contact $R^{\prime}$ were varied. Variation of $R^{\prime}$ was done in order to obtain various values of the maximum Hertzian pressure of $0.5,1.0,2.0$ and $3.0 \mathrm{GPa}$. As a first guess, three parameters can be suggested to represent vapour bubble formation potential. These are the minimum pressure, the pressure gradient, and the integral over all sub-ambient pressures. The minimum pressure by itself is not likely to be enough. Also a dynamic aspect and a cumulative effect are important.

The dynamic aspect would be represented by the pressure gradient and the cumulative aspect by the integral over all sub-ambient pressures. Generally the pressure drop in the exit region takes place with a large negative pressure gradient. Approximating its absolute maximum value accurately for high loads would require very small local mesh sizes in the numerical solution process. Therefore, in the present pilot study we restricted ourselves to the minimum pressure and the integral over all sub-ambient pressures.

The results of the parametric study are presented in Fig. 3 . This figure shows that the minimum pressure itself is relatively insensitive to the velocity and load. In fact, with increasing velocity it appears to approach an asymptotic value, a subject which requires further investigation. The value of the integral over all sub-ambient pressures varies more for the different loading cases. This can be explained as follows. With increasing velocity the magnitude of the pressure drop in the outlet region remains roughly the same. However, the drop itself, and the following build up to ambient pressure are much more gradual, i.e. with smaller gradients, which lead to a larger value of the integral over the sub-ambient pressures.

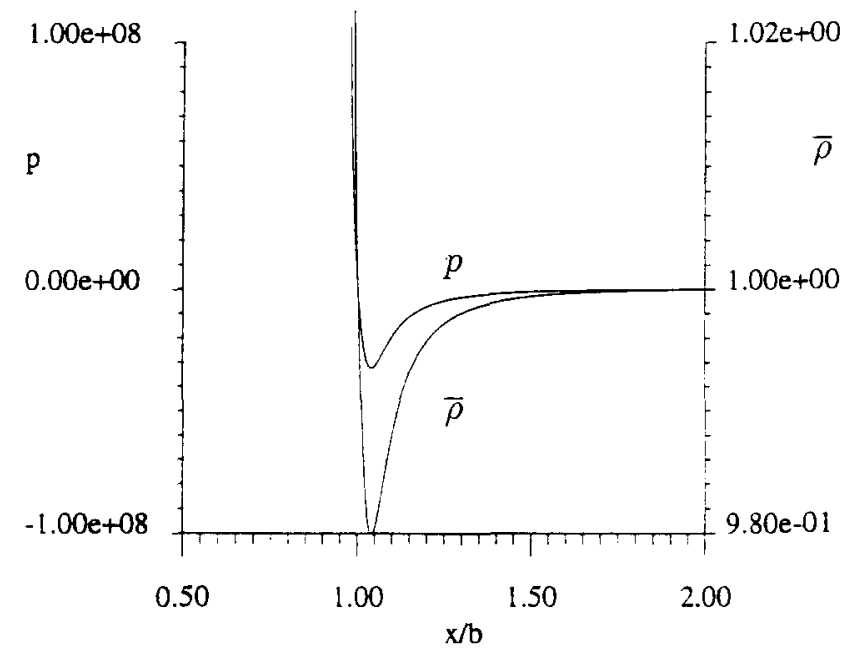

Fig. 2. Pressure profile $p$ and relative lubricant density in the sub-ambient pressure region of the solution shown in Fig. 1. 

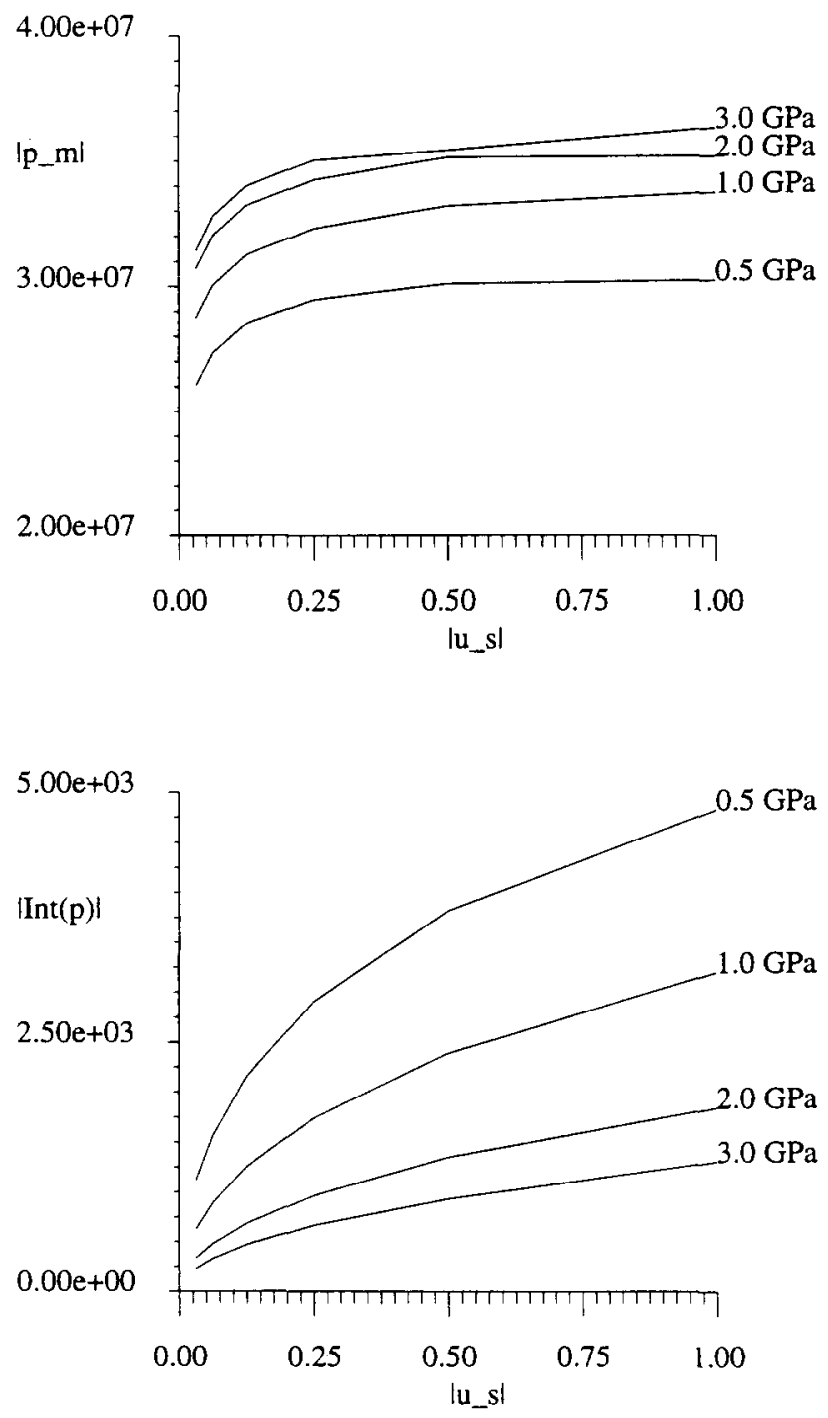

Fig. 3. Minimum pressure (top), and integral over all sub-ambient pressures (bottom) as a function of sum velocity for maximum Hertzian pressures of $0.5,1.0,2.0$ and $3.0 \mathrm{GPa}$.

This above may indicate that the integral over the subambient pressures provides a better criterion to discriminate between situations for the local vapour bubble formation and cavitation damage potential. However, this can at best be partly true. For example based upon Fig. 3 one might con-

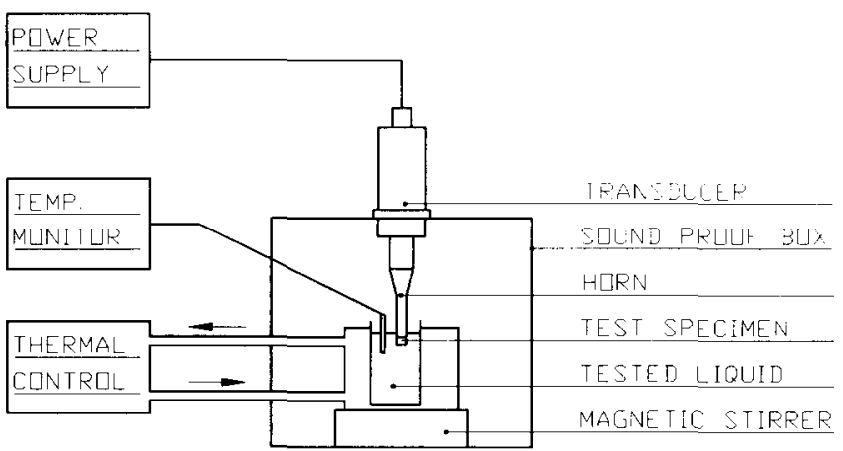

Fig. 4. Schematic diagram of vibratory cavitation erosion apparatus. clude that the risk of cavitation damage increases with incrcasing velocity. Furthermore the risk would decrease with increasing load. This latter conclusion seems to contradict what one would expect. If however the pressure gradients were monitored one would probably arrive at the opposite conclusion, as the steepness of the pressure curve in the exit region increases with increasing load $[10,11]$. Hence, a measure of bubble formation potential, or cavitation damage risk should also include the effect of the pressure gradient.

\section{Experimental approach}

\subsection{Experimental equipment and test conditions}

\subsubsection{Scope}

The experiments were carried out with an ultrasonic oscillator (Fig. 4) operating at a frequency of $20 \mathrm{kHz}$ with a maximal power output of $400 \mathrm{~W}$. The test specimen was mounted at the tip of an exponential oscillator (horn) and immersed to a depth of $20 \mathrm{~mm}$ from the surface of the tested liquid. During the experiment the test specimen oscillated in a sinusoidal motion, at a constant amplitude of $73 \mu \mathrm{m}$.

During the upward motion of the specimen, low pressure is formed in the liquid, causing it to cavitate. Upon the downward motion of the specimen, high pressure is formed in the liquid, causing the cavitation bubbles to implode. Implosion of the bubbles very near and in contact with the surface, cause repeated high thermal and mechanical loading, which result in erosion.

\subsubsection{Specimen}

The test specimens (Fig. 5) were prepared from aluminium $51 \mathrm{ST}$, which is the equivalent of the U.S. alloy 6082 and DIN alloy A1MgSi 1.0. Its outer diameter is $12.7 \mathrm{~mm}$, identical to that of the horn tip. The mechanical properties of the tip material and its nominal composition are presented in Appendix $\mathrm{C}$. When using flat shaped specimens in several oil cavitation tests, it was observed that part of the bubbles flowed in parallel to the specimen flat surface, thus causing the following phenomena:

(a) agitation of the oil surface;

(b) inhomogeneous erosion of the specimen, mainly along radial lines.

Changing to a cup shaped specimen deflected the flow to the longitudinal direction, thereby avoiding liquid agitation and increasing erosion pattern homogeneity.

\subsubsection{Liquids}

The cavitation erosion tests were conducted in a variety of lubricating liquids, including: straight mineral oils, mineralbased oils, biodegradable oils and synthetic liquids. These liquids have an absolute (kinematic) viscosity in the range of $9-460 \mathrm{~mm}^{2} \mathrm{~s}^{-1}$ ) at $40^{\circ} \mathrm{C}$. A few properties of the tested liquids are listed in Appendix D. 


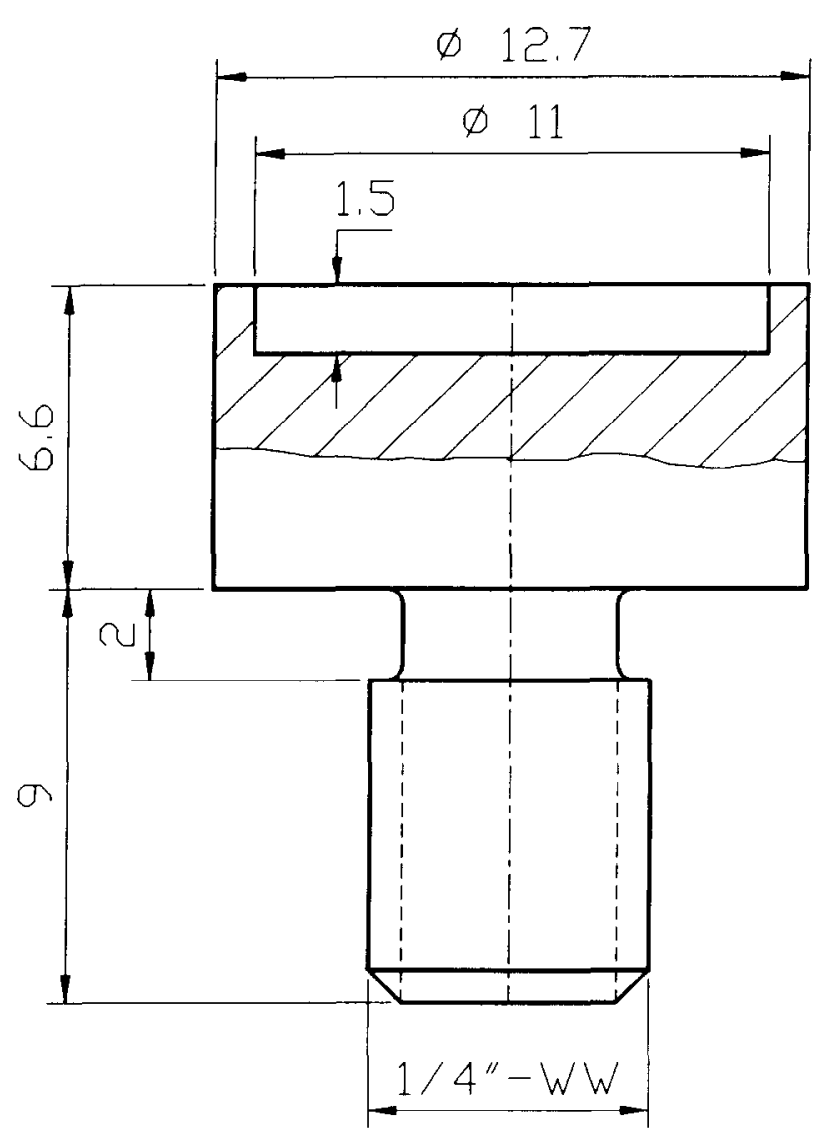

Fig. 5. Cup-shaped test specimen.

\subsubsection{Temperature}

The temperature of the liquids during the tests was maintained at a nominal value of $40{ }^{\circ} \mathrm{C}$. This was achieved by means of a thermostatic bath, controlling the temperature of the water jacket and a magnetic stirring device for the tested liquid. This temperature was chosen for two main reasons:

(a) cavitation erosion in lubricants increases as a function of temperature within this range [6]. Hence, testing at $40{ }^{\circ} \mathrm{C}$, instead of $25^{\circ} \mathrm{C}$ as required in ASTM G32-92 [12], will increase the erosion rate and shorten the test duration;

(b) the maximum allowed working temperature for the transducer assembly is $50^{\circ} \mathrm{C}$.

\subsubsection{Amplitude and power input}

Tip amplitude is kept constant by the power supply, regardless of the acoustic load. This means that for a given amplitude setting it will remain constant while operating in a variety of liquids. Monitoring the power input during the tests revealed the following variations:

(a) at the initial phase of the test (about $15 \mathrm{~min}$ ), variation was largest, reaching a maximum value of $\pm 12.5 \%$

(b) during the rest of the time variation decrcascd appreciably, reaching the maximum value of $\pm 3 \%$.

These observations indicate that the larger power input variation, which appears at the initial stage, may be due to gas release from the liquid simultaneously with vapour cav- itation. After stabilization of the gas content in the liquid, power input remains highly stable. The second conclusion from this observation is that under cavitation conditions with this equipment it is possible to get both amplitude and power input simultaneously at a constant level. Thus it is possible to settle the dispute between those who advocate testing with constant amplitude and those who support testing with constant power input [13].

\subsubsection{Duty cycle}

Cavitating a liquid in this equipment can be achieved by two modes: continuous or pulsed. In the pulsed mode the tip vibrates only part of each operation cycle, according to the duty cycle setting. For these tests the pulsed mode was chosen, with a duty cycle of $50 \%$. This means that during each second of equipment operation the tip vibrates only for $0.5 \mathrm{~s}$. Operation in this mode enables introducing a power level close to the maximum value with less heating of the fluid. The obvious disadvantage of this procedure is the doubling of the overall test duration. An interesting point in favour of intermittent cavitation exposure is that it better simulates actual operation conditions of machine elements such as: gears, rolling contact bearings and dynamically loaded journal bearings.

Examining a surface element in these items, while operating under cavitation conditions, reveals that it is exposed to cavitation at a certain frequency. This frequency coincides with the pressure fluctuations caused by periodic contact (in gears and rolling contact bearings) and by transverse motion of the journal within the bearing.

\subsubsection{Gravimetry}

Cavitation erosion was measured by the tip's mass loss, after every $30 \mathrm{~min}$ of net exposure to cavitation. The specimens were dismantled from the horn, cleaned in a solvent and weighed on an electronic balance, having a resolution of $10^{-5} \mathrm{~g}\left(10^{-2} \mathrm{mg}\right)$.

\subsubsection{Test duration}

The net test duration was $2 \mathrm{~h}$. This duration was chosen because it is longer than the incubation time for the specimen material and enables distinct classification of the liquids. Longer test periods would increase the damage, but have little practical significance as the equipment having that level of mass loss would be severely damaged or even inoperable by then.

\subsection{Test results}

\subsubsection{Scope}

Gravimetric results for the tested oils are shown in Figs. 6-9 for the net cxposure time to cavitation conditions of $2 \mathrm{~h}$. These results are grouped according to oil type, and designated as shown in Appendix D. The results for each oil were tested by linear regression and found to have a high degree of linearity. 
MASS LOSS (mg)

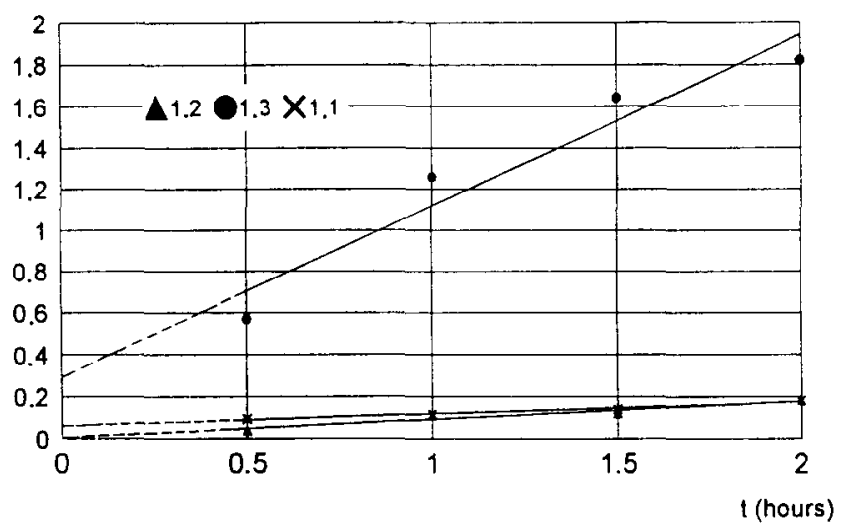

Fig. 6. Cavitation erosion in straight mineral oils. Note: for oils designations in Figs. 6-11, see Appendix D.

\section{MASS LOSS (mg)}

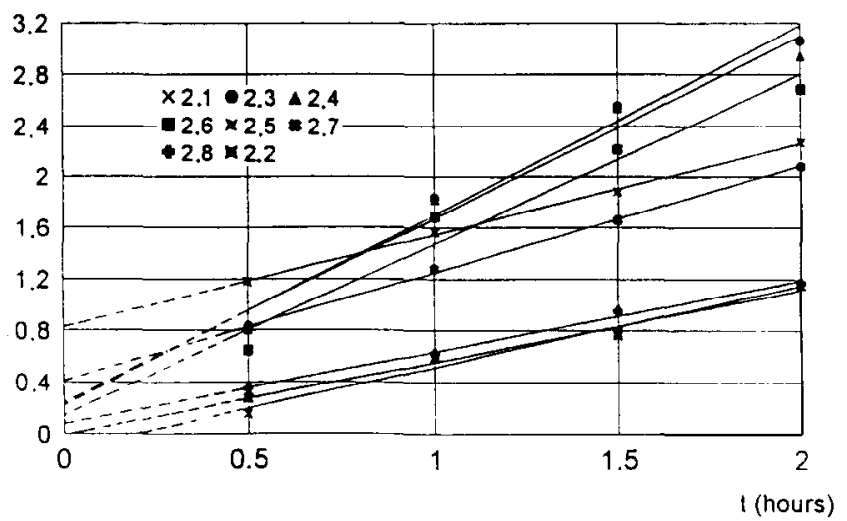

Fig. 7. Cavitation erosion in mineral-based oils.

\section{MASS LOSS (mg)}

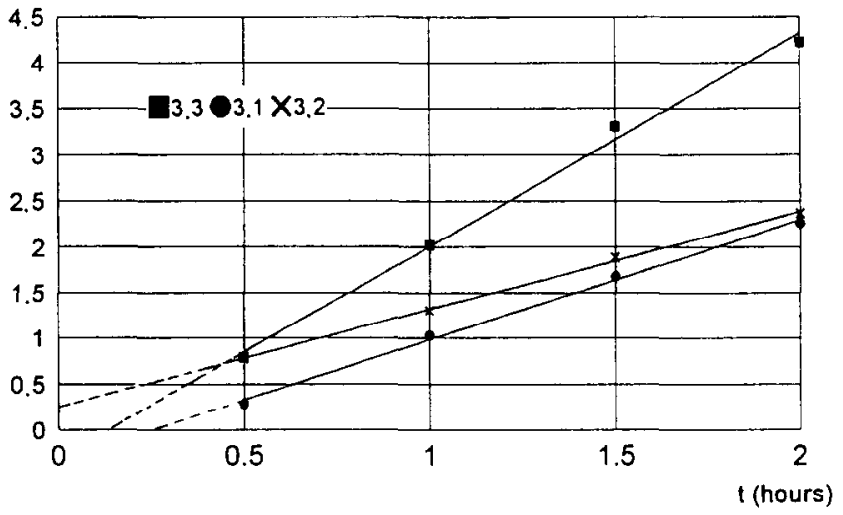

Fig. 8. Cavitation erosion in biodegradable oils.

Regression coefficients were found to be in the range of $0.933-0.999$, with an average value of 0.988 . The repeatability of the test method was evaluated for two oils,
Cassida-HF 46 and Turbo-T68, each one tested twice. Gravimetric results of these tests shown on Fig. 10 indicatc a relative difference at the end of the tests of 0 and $4.2 \%$ respectively. The number of these repetitive tests is rather small, but they serve as a first approximation for the repeatability of

MASS LOSS (mg)

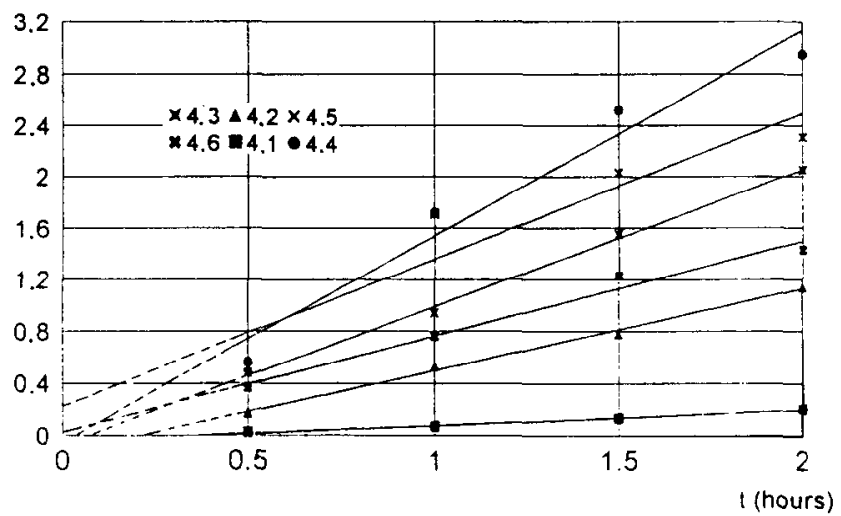

Fig. 9. Cavitation erosion in synthetic lubricants.

MASS LOSS (mg)

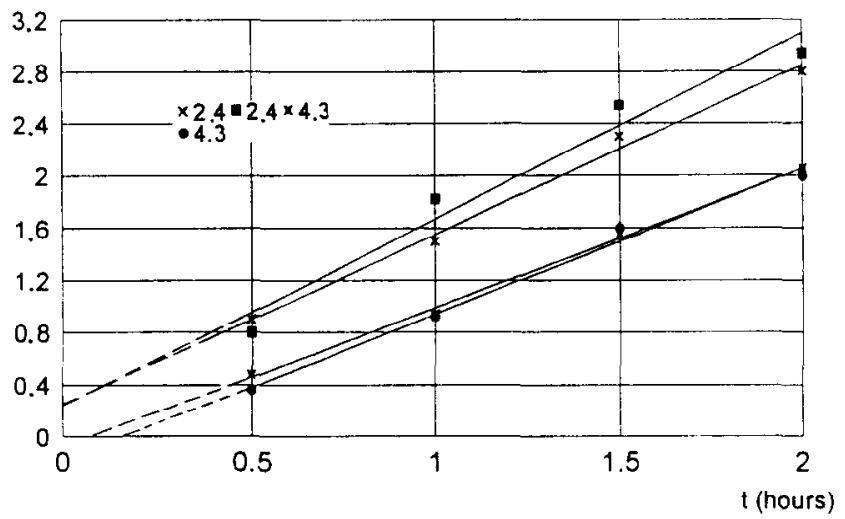

Fig. 10. Repeatability tests with two lubricants.

MASS LOSS (mg)

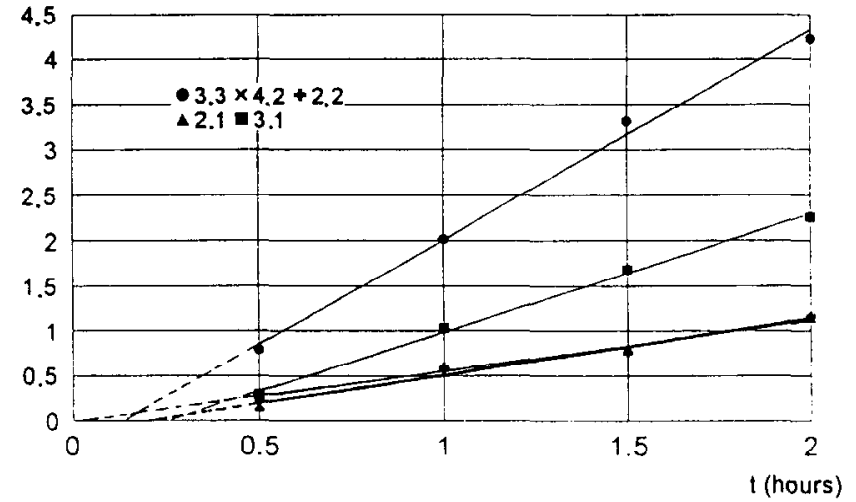

Fig. 11. Oil type effect with lubricants having the same absolute viscosity. 
this method. It is worth mentioning that the expected repeatability in erosion tests in water, according to ASTM standard $\mathrm{G} 32-85$, is in the order of $20 \%$.

\subsubsection{Viscosity}

Some cavitation investigators claim that the viscosity of a lubricant is a dominant property in its erosion capability. A typical example of such studies is that performed by Vercon and Rac [6]. The results of this investigation do not support Vercon's claim. From Figs. 7-9, which describe the cavitation erosion in various lubricants belonging to three types, it is evident that there is no direct correlation between the viscosity and the cavitation erosion in these liquids. This conclusion can be drawn from Fig. 11, too, in which the cavitation erosion in five lubricants, having the same kinematic viscosity at $40^{\circ} \mathrm{C}$, can be compared.

Cavitation is caused by the application of negative pressures in a liquid. This pressure, causing tension in the liquid, may be induced by flow, acoustically or mechanically [14] Hence, it seems that the dominant liquid property in the cavitation process is its tensile strength. It therefore seems important to study this property more carefully.

\subsubsection{Classification criteria}

Several parameters were considered for the evaluation of cavitance of lubricating oils:

(a) cavitation threshold;

(b) cavitation power input;

(c) maximum cavitation erosion;

(d) slope of cavitation erosion line.

\section{(a) Cavitation threshold}

The correlation of this parameter with cavitation erosion is low. This may be due to the possibility that cavitation inception will occur when a nucleus of critical size is present in the liquid and may not be an indication to the quantity of these nuclei. Whereas cavitation erosion, which is a cumulative process, seems to be affected by both size and quantity of the critical nuclei. Accordingly, cavitation threshold was discarded as a criterion for cavitance.

\section{(b) Cavitation power input}

During the exposure of a specimen to cavitation, on the test equipment used, it is possible to medsure the power input into the cavitating fluid. Comparing these values for the var-

Table 1

Classification chart for the tested liquid lubricants

\begin{tabular}{|c|c|c|c|c|c|}
\hline Cavitance & & 1 & 2 & 3 & 4 \\
\hline Rating & $\mathrm{CE}_{2}(\mathrm{mg})$ & Straight mineral oils & Mineral oil with additives & Biodegradable hydraulic oils & Synthetic-based oils \\
\hline \multirow[t]{3}{*}{ Medium low } & 2 & & $\begin{array}{l}\text { Turbo-T68 } \\
\text { Tonna-T68 }\end{array}$ & & \\
\hline & & & & & Cassida-GL150 \\
\hline & & & & Naturelle-HFE46 & \\
\hline \multirow{5}{*}{$\begin{array}{l}\text { Medium } \\
\text { high }\end{array}$} & 1 & Vitrea-150 & & & \\
\hline & & & & & Cassida-GL460 \\
\hline & & & Omala-320 & & \\
\hline & & & Tellus-32 & & \\
\hline & & & Tellus-T32 & & Cassida-HF32 \\
\hline \multirow[t]{2}{*}{ High } & & Ondina-15 & & & \\
\hline & & Vitrea-9 & & & Cassida-HF15 \\
\hline
\end{tabular}


ious liquids revealed no systematic trend nor correlation to the gravimetric results. Hence, the cavitation power input was discarded as a cavitance parameter.

\section{(c) Maximum cavitation erosion}

This parameter $-\mathrm{CE}_{2}-$ is defined as the cumulative mass loss at the termination of the test, after $2 \mathrm{~h}$. The highest $\mathrm{CE}_{2}$ in the tested oils is $4.322 \mathrm{mg}$ for fluid BD-32 and the lowest corresponding value for Vitrea- 9 is $0.173 \mathrm{mg}$. The ratio between these extreme values is nearly 25 , which covers a large range, practical for engineering application. It is obvious that a low value of $\mathrm{CE}_{2}$ corresponds to a high degree of cavitance and vice versa.

This parameter is easy to measure and enables liquid classification, hence it was chosen as a classifying criterion. Values of mean depth penetration in micrometres can be obtained by multiplication of the mass loss values in milligrams by 2.076 .

Glycerin, which is a defined chemical $\left(\mathrm{C}_{3} \mathrm{H}_{8} \mathrm{O}_{3}\right)$, having a viscosity comparable with those of lubrication oils, was chosen as a reference material for these tests. The $\mathrm{CE}_{2}$ value for glycerin reached $93.66 \mathrm{mg}$, which is over 20 times greater than the highest value measured fro the tested lubricants.

\section{(d) Slope of cavitation erosion line}

This paramcter- $a$-is defined as the slope of the line $y=a x+b$, which describes the cavitation erosion as a function of the exposure time. The highest slope in the tested oils is $2.316 \mathrm{mg} \mathrm{h}^{-1}$ for fluid BD-32 and the lowest corresponding value for Vitrea-9 is $0.0568 \mathrm{mg} \mathrm{h}^{-1}$. The ratio between these extreme values is nearly 40 , which defines a larger range than that for $\mathrm{CE}_{2}$. However, due to the fact that $b$ is not constant, there is no full correlation between values of $a$ and $\mathrm{CE}_{2}$, hence, this parameter was discarded as a cavitance criterion.

\subsubsection{Classification chart}

The classification chart, Table 1, includes all the lubricants tested, each listed in its group and according to its $\mathrm{CE}_{2}$ value. This chart portrays a clear picture of the relative standing of all the tested lubricants and enables its use as an engineering tool in the following ways:

\section{(a) Lubricant selection}

After selecting a proper group of lubricants for a certain application, an adequate oil within that group may be selected according to its cavitance.

\section{(b) Lubricant change}

After detection of cavitation erosion in a hydraulic system, a proper lubricant can be chosen with a higher cavitance.

It should be emphasized, however, that the data in this chart are based on commercial oils tested with an aluminium tip by means of a vibratory generator under certain conditions. This chart therefore manifests the possibility of preparing a valuable engineering tool, but the technical data require extension and further verification.

\section{Conclusions}

(1) Using a simple EHD line contact problem as a model, a first attempt was made to obtain a theoretical tool for use in cavitation damage control in lubricated contacts. For this purpose the sub-ambient pressure and the integral over the region of sub-ambient pressures generated in the exit zone were monitored as a function of the operating conditions. It was shown that when looking for a measure of local vapour bubble formation potential, the latter integral will provide sufficient discrimination between different load conditions. However it was also shown that such an integral by itself is not sufficient and that pressure gradients should be studied too.

(2) Lubricating liquids can be classified according to their cavitance. A reasonable classification criterion for this purpose is the cumulative cavitation erosion of an aluminium specimen after $2 \mathrm{~h}$ exposure to controlled cavitation conditions, induced by vibration equipment. The resulting classification chart of 20 oils, belonging to four groups, may be used as a preliminary guide for lubricant selection in cavitating systems.

(3) No direct correlation has been found in this investigation between the viscosity of the lubricant and the cavitation erosion obtained with it. As cavitation is caused mainly by the application of tensile stress in liquids, it is postulated that the tensile strength of lubricants is a dominant property controlling their cavitance.

(4) Further research is required in the following directions:

(a) investigate sub-ambient pressures caused by squeeze effect and by large local surface features;

(b) correlate pressure-based criteria, such as minimum pressure, pressure gradient and pressure integral, to the actual cavitation erosion;

(c) extend cavitation erosion tests with additional lubricants and tip materials;

(d) explore the effects of lubricant contamination on cavitation erosion.

These complimentary investigations will contribute to the preparation of a comprehensive scientific concept and a reliable engineering tool.

\section{Acknowledgements}

The authors wish to acknowledge Shell Co. for supplying the test lubricants, technical information and guidance. 


\section{Appendix A. Nomenclature}

$b \quad$ radius of Hertzian contact, $b=\sqrt{8 w R^{\prime} / \pi E^{\prime}}$

$\mathrm{CE}_{2} \quad$ cumulative weight loss after $2 \mathrm{~h}$ exposure to cavitation conditions

E modulus of elasticity

$E^{\prime} \quad$ reduced modulus of elasticity, $2 / E^{\prime}=(1-$ $\left.\nu_{1}^{2}\right) / E_{1}+\left(1-\nu_{2}^{2}\right) / E_{2}$

$G \quad$ dimensionless materials parameter $G=\alpha E^{\prime}$

$h \quad$ film thickness

$H \quad$ dimensionless film thickness, $H=h R_{x} / b^{2}$

$L \quad$ dimensionless material parameter (Moes), $L=G(2 U)^{0.25}$

$M \quad$ dimensionless load parameter (Moes), $M=$ $W(2 U)^{-0.5}$

$p \quad$ pressure

$p_{\mathrm{h}} \quad$ maximum Hertzian pressure, $p_{\mathrm{h}}=2 w / \pi b$

$P \quad$ dimensionless pressure, $P=p / p_{\mathrm{h}}$

$R \quad$ radius of curvature

$R^{\prime} \quad$ reduced radius of curvature, $1 / R^{\prime}=1 / R_{1}+$ $1 / R_{2} u$

surface velocity

sum velocity, $u_{\mathrm{s}}=u_{1}+u_{2}$

dimensionless speed parameter, $U=$ $\eta_{\mathrm{o}} u_{\mathrm{s}} /\left(2 E^{\prime} R_{x}\right)$

$w \quad$ external load per unit width

$W \quad$ dimensionless load parameter, $W=w /\left(E^{\prime} R\right)$

$x, x^{\prime} \quad$ coordinate

$x_{\mathrm{a}}, x_{\mathrm{b}} \quad$ boundaries of the domain

$\alpha \quad$ pressure viscosity index

$\alpha \quad$ dimensionless parameter, $\alpha=\alpha p_{\mathrm{h}}$

$\lambda$ dimensionless speed parameter, $\lambda-$

$6 \eta_{\mathrm{o}} u_{\mathrm{s}} R_{x}^{2} / \mathrm{b}^{3} p_{\mathrm{h}}$

$\eta \quad$ viscosity

$\eta_{\mathrm{o}} \quad$ viscosity at ambient pressure

$\rho \quad$ density

$\rho_{\mathrm{o}} \quad$ density at ambient pressure

dimensionless density, $=\rho / \rho_{\mathrm{o}}$

$\nu \quad$ Poisson's ratio

Subscripts

1,2 surface/body

0 constant

m minimum

c central, at $\partial p / \partial x=0$
Appendix B. Bearing loading conditions-various parameters and their values

\begin{tabular}{lll}
\hline Parameter & Value & Dimension \\
\hline$E^{\prime}$ & $2.26 \times 10^{11}$ & {$[\mathrm{~Pa}]$} \\
$\alpha$ & $2.2 \times 10^{-8}$ & {$\left[\mathrm{~Pa}^{-1}\right]$} \\
$\eta_{0}$ & $40.0 \times 10^{-3}$ & {$[\mathrm{~Pa} \mathrm{~s}]$} \\
$R$ & $1.41 \times 10^{-2}$ & {$[\mathrm{~m}]$} \\
$u_{\mathrm{s}}$ & 0.5 & {$\left[\mathrm{~m} \mathrm{~s}^{-1}\right]$} \\
$b$ & $5 \times 10^{-4}$ & {$[\mathrm{~m}]$} \\
$h_{\mathrm{m}}$ & 0.118 & {$[\mu \mathrm{m}]$} \\
$h_{\mathrm{c}}$ & 0.145 & {$[\mu \mathrm{m}]$}
\end{tabular}

Dimensionless parameters

$\begin{array}{ll}M & 68.4\end{array}$

$\begin{array}{ll}L & 6.7\end{array}$

$\begin{array}{ll}\alpha & 22.0 \\ \lambda & \end{array}$

$\lambda \quad 7.94 \times 10^{-4}$

$\begin{array}{ll}W & 1.19 \times 10^{-4} \\ U & 1.51 \times 10^{-12} \\ G & 5060\end{array}$

Appendix C. Specimen tip material compostion and properties

\begin{tabular}{lll}
\hline Property & Unit & Value \\
\hline Density & $\mathrm{kg} \mathrm{m}^{-3}$ & 2700 \\
\hline Yield strength & $\mathrm{MN} \mathrm{m}^{-2}$ & $240-380$ \\
Tensile strength & $\mathrm{MN} \mathrm{m}^{-2}$ & $320-380$ \\
Elongation & $\%$ & $10-15$ \\
Hardness & Brinell & $90-100$
\end{tabular}

Designation: 51 ST (US 6082; DIN AlMgSi 1.0). Composition: Si $1.0 \%, \mathrm{Mg} 0.6 \%$.

\section{Appendix D. Tested lubricants}

\begin{tabular}{|c|c|c|c|c|}
\hline Tested liquid & $\begin{array}{l}\text { Kinematic viscosity } \\
\text { at } 40^{\circ} \mathrm{C} \\
\left(\mathrm{mm}^{2} \mathrm{~s}^{-1}\right)\end{array}$ & $\begin{array}{l}\text { Viscosity } \\
\text { index }\end{array}$ & $\begin{array}{l}\text { Specific gravity } \\
\left(\mathrm{kg} \mathrm{l}^{-1}\right)\end{array}$ & Notes \\
\hline \multicolumn{5}{|c|}{ 1. Straight mineral oils } \\
\hline 1.1. Vitrea-9 & 8.8 & 50 & 0.868 & Paraffinic type oils \\
\hline 1.2. Ondina-15 & 15 & & 0.853 & \\
\hline
\end{tabular}


1.3. Vitrea- 150

2. Mineral-based oils

2.1. Tellus-32

2.2. Tellus-T32

2.3. Tellus- 68

2.4. Turbo-T68

2.5. Hydrol-HV46

2.6. Tonna-TX68

2.7. Tonna-TX220

2.8. Omala-320

3. Biodegradable hydraulic oils

3.1. Naturelle-HFR 32

3.2. Naturelle-HFE 46

3.3. Fluid-BD 32

4. Synthetic oil based

4.1. Cassida-HF 15

4.2. Cassida-HF 32

4.3. Cassida-HF 46

4.4. Cassida-HF 68

4.5. Cassida-GL 150

4.6. Cassida-GL 460
97

$98 \quad 0.870$

$181 \quad 0.875$

$97 \quad 0.880$

$98 \quad 0.876$

$180 \quad 0.880$

$108 \quad 0.876$

$110 \quad 0.890$

$\begin{array}{ll}100 & 0.899\end{array}$

180

$120 \quad 0.819$

$\begin{array}{ll}140 & 0.829\end{array}$

$140 \quad 0.833$

$\begin{array}{ll}140 & 0.837\end{array}$

$140 \quad 0.842$

$155 \quad 0.849$
Universal extreme pressure type, hydraulic and gear oils

\section{References}

[1] H.G. Elrod, A cavitation algorithm, ASME J. Tribol., 103 (1981) 350 354.

[2] C.M. Woods, and D.E. Brewe, The solution of the Elrod algorithm for a dynamically loaded journal bearing using multigrid techniques, ASME J. Tribol., IIl (1989) 302-308.

[3] R. Garcia, and F.G. Hammitt, Cavitation damage and correlations with material and fluid properties, J. Basic Eng., (Dec. 1967) 753763.

[4] A. Thiruvengadam, Role of physical properties of liquids in cavitation erosion, National Bureau of Standards Spec. Publication 394, 1974.

[5] J.M. Hobbs and D. Rachman, Environmentally Controlled Cavitation Test, ASTM STP 474, 1970, p 29-47.

[6] I.J. Vercon and A. Rac, Influence of lubricating oil on cavitation erosion damage of engine bearing materials, Proc. Eurvtrib-81, Vol. III, p. 317-323.

[7] F.T. Barwell and D. Scott, Effect of lubricant on pitting failure of ball bearings, Engineering, 6 (1956) 9-12.
[8] D. Dowson, and G.R. Higginson, Elastohydrodynamic Lubrication, the Fundamentals of Roller and Gear Lubrication, Pergamon, Oxford, 1966.

[9] C.J.A. Roelands, Correlational aspects of the viscosity-temperaturepressure relationship of lubricating oils, Ph.D. Thesis, Technical University of Delft, The Netherlands, 1966. (V.R.B. Groningen, The Netherlands).

[10] C.H. Venner, W.E. ten Napel, and R. Bosma, Advanced multilevel solution of the EHL line contact, ASME J. Tribol., 112 (1990) 426432.

[11] C.H. Venner, Multilevel solution of the EHL line and point contact problem, Ph.D. Thesis, University of Twente, Enschede, The Netherlands, 1991, ISBN 90-9003974-0.

[12] Standard Method of Vibratory Cavitation Erosion Test, G32-92, ASTM, Philadelphia, PA, 1992.

[13] B.C.S. Rao and D.H. Buckley, Erosion of aluminum 6061-T6 under cavitation attack in mineral oil and water, Wear, 105 (1985) 171-182.

[14] T.G. Leighton, The Acoustic Bubble, 1994 ISBN 0-12-441920-8, p. 73-75. 